\title{
MODELIZACIÓN DE LA MOVILIDAD EN LOS RANKINGS DE HOTELES
}

\author{
José-Luis Ximénez-de-Sandoval* \\ The American College in Spain \\ Antonio Fernández-Morales** \\ Antonio Guevara-Plaza*** \\ Universidad de Málaga
}

\section{RESUMEN}

El ranking de hoteles de TripAdvisor, ha sido analizado desde múltiples perspectivas, pero son pocos los investigadores que han estudiado su evolución temporal. Este artículo presenta uno de los primeros trabajos sobre la movilidad en los rankings de hoteles. Distinguimos entre rankings líquidos, y sólidos, definimos la Movilidad de un ranking, distinguiendo entre micro y macromovilidad. Además, introducimos el concepto de vector trayectoria como objeto central del análisis. Por último, presentamos un modelo para medir la movilidad, que se aplicará a una muestra de 771 hoteles de ciudades españolas.

Palabras clave: Ranking; movilidad; hoteles; TripAdvisor; minería de datos; reputación online.

\section{Modelling hotel ranking mobility}

\section{ABSTRACT}

Many researchers have studied from different perspectives the TripAdvisor's Hotel Rankings. But only few have analyzed how it evolves over time. This paper presents one of the first studies on mobility in hotel rankings. We define the concepts of liquid and solid

Fecha de recepción: 6 de octubre de 2017.

Fecha de aceptación: 30 de mayo de 2018.

* The American College in Spain, an International Center of Broward College, Florida, USA. Urb. La Alzambra. 29660 Marbella. MÁLAGA (España).E-mail: joseluis.xs@uma.es

** Facultad de Ciencias Económicas. Universidad de Málaga. C/ Ejido, 6. 29071. MÁLAGA (España). E-mail: afdez@uma.es

*** Facultad de Turismo. Universidad de Málaga. C/ León Tolstoi, s/n. 29071. MÁLAGA (España). E-mail: guevara@uma.es 
rankings, mobility, as well as micro and macromobility. We also introduce the concept of Path Vector as the main object of the analysis. Finally, we present a model to measure mobility, which will be applied to a sample of 771 hotels in Spanish cities.

Keywords: Hotel Ranking Mobility; TripAdvisor; Online reviews; User generated content; Data mining; Online reputation; e-wom.

\section{INTRODUCCIÓN}

La gran influencia que los rankings de hoteles ejercen sobre los clientes cuando estos van a elegir el establecimiento donde alojarse es reconocida por expertos en la materia, académicos y profesionales (Ghose et al.2012). También los empresarios se ven afectados por estas clasificaciones en la medida en que una buena o mala posición puede influir no sólo en su reputación sino en el número de reservas y por tanto en la cifra de negocio (Callarisa-Fiol et al. 2012).

No obstante, y pese a la revolución que han supuesto para el sector turístico compañías como TripAdvisor o Booking (Xiang y Gretzel, 2010; Ong, 2012), la movilidad de estos rankings, ha sido muy poco estudiada en la literatura científica. Las más recientes revisiones bibliográficas (Kwok et al. 2017; Sotiriadis y Sotiriadis, 2017; Chen y Law, 2016) dan buena muestra de ello al no mencionar ningún trabajo que se haya ocupado en estos últimos años de analizar la variación a lo largo del tiempo de las posiciones que ocupan los hoteles en la clasificación.

Sin embargo, creemos que el estudio de la evolución de estos rankings (de hoteles así como de restaurantes) es de gran importancia tanto para empresarios como para clientes, dada la gran influencia que ejercen sobre todos ellos.

Aunque a lo largo de este artículo analizaremos a fondo las implicaciones que la movilidad de los rankings tiene para los distintos protagonistas de la industria turística, pensemos por un momento en una clasificación de hoteles o restaurantes que permanece prácticamente invariable a lo largo del tiempo. ¿Seguirían tan preocupados los hoteleros en mejorar su posición si supieran que el puesto que ocupan en la actualidad apenas iba a variar en el futuro? ¿Estarían motivados los usuarios a escribir comentarios sobre un hotel si fueran conscientes de que su opinión, tanto positiva como negativa, no iba a influir en la posición que ocupa el hotel en el ranking?

En este caso, deberíamos plantearnos como expertos lo que habría que responder a un hotelero que nos pidiera consejo para lograr que su hotel llegase a estar entre los mejores de la ciudad en el menor tiempo posible. Tendríamos que contestarle que debido a la mínima movilidad que presenta el ranking de hoteles en su ciudad, sus posibilidades de estar algún día entre los mejores son mínimas, y que del mismo modo que lleva dos o tres años en la zona media/baja del ranking, la posición que ocupa en la clasificación apenas variará a medio plazo.

En sentido contrario, podríamos pensar en una clasificación donde los hoteles que hace unos meses estaban entre los mejores de la ciudad, estuvieran ahora situados en la zona 
más baja, y aquellos que estaban en las peores posiciones, estén ahora en los puestos más altos. ¿Qué sensación causaría en un cliente ver que el hotel elegido para sus próximas vacaciones, que hace unos días estaba entre los mejores, se encuentra ahora situado en la zona baja del ranking?

Acabamos de ver dos ejemplos, quizás extremos, que nos ilustran sobre la necesidad de estudiar en profundidad la dinámica de los rankings.

El principal objetivo de este trabajo no es tanto responder a las preguntas anteriores, sino despertar el interés de la comunidad científica, así como de empresarios y expertos en turismo, sobre este aspecto tan desconocido de los rankings, que debe considerarse como una de las características fundamentales de los mismos. En definitiva, queremos dar a conocer una nueva línea de investigación en turismo apenas explorada hasta la fecha y centrada en el estudio de la movilidad en las posiciones que los hoteles (y restaurantes) ocupan en la clasificación de TripAdvisor.com y Booking.com en destinos de todo el mundo.

El segundo objetivo es presentar los principales conceptos, definiciones y términos para contribuir a la creación de un marco conceptual que facilite el desarrollo de futuros estudios en esta nueva materia.

Como tercer objetivo, nos proponemos crear un modelo que permita cuantificar la movilidad de un ranking y en definitiva analizar su evolución temporal. En este sentido realizamos una innovadora aproximación teórica al concepto de movilidad aportando una novedosa metodología que permite calcular de forma eficiente la movilidad de los rankings de hoteles, a través de un modelo teórico que define el ranking como un sistema dinámico en el que sus elementos van cambiando de posición a lo largo del tiempo.

Además, Este trabajo quiere aportar luz sobre el funcionamiento de los rankings de hoteles, en especial de aquellos más reconocidos por turistas y hoteleros, TripAdvisor y Booking, porque como señalan Scott y Orlikowski, (2012), TripAdvisor está configurado de tal manera que produce una forma de trasparencia para los comentarios de los turistas mientras que oscurece el mecanismo que ordena esos comentarios. Así, por una parte, estas plataformas son consideradas transparentes al poner a disposición del usuario final una inabarcable cantidad de información en distintos formatos (puntuaciones, comentarios, fotografías), con valoraciones tanto positivas como negativas aportadas por otros usuarios que resultan de gran utilidad a la hora de seleccionar un establecimiento. Además, sus diseños permiten que mediante diversos filtros (por puntuación, por tipo de viajero, por época del año o por idioma), el usuario pueda acceder con suma facilidad a millones de datos.

Pero al mismo tiempo que se pone a disposición del usuario toda esta información, se están ocultando los mecanismos utilizados para ordenar esos rankings. Más allá de unas leves nociones sobre el funcionamiento del algoritmo encargado de la configuración del ranking (TripAdvisor, 2016), no podemos conocer con claridad cómo se establece la valoración final del hotel y por tanto su posición en la clasificación. El gran valor de TripAdvisor como creador de rankings no está en su algoritmo, sino en su capacidad de atraer a cientos de miles de usuarios de todo el mundo para que compartan sus opiniones, valoraciones y experiencias. Por eso entendemos que esta opacidad no está en ningún caso justificada, dando argumentos a quienes sospechan de comportamientos poco éticos en la elaboración de estos rankings. 
Sin poner en ningún momento en duda la honestidad y rigor de quienes diseñan y gestionan estos rankings, no es menos cierto que dada su reconocida falta de trasparencia (Mellinas et al., 2015) y la creciente influencia que ejercen en la industria hotelera (Mateos et al., 2015), resulta sorprendente que hasta el momento no exista ningún tipo de supervisión o control por parte de instituciones públicas o privadas sobre los mismos, que auditen y verifiquen su correcto funcionamiento. Por todo ello, creemos que es obligación del investigador, tratar de averiguar cómo funcionan, y detectar y alertar sobre posibles ineficiencias o anomalías en su comportamiento.

Para concluir esta introducción, indicamos brevemente cómo se organiza el presente documento. En primer lugar, realizaremos una revisión bibliográfica sobre la movilidad, los rankings y TripAdvisor. Debido a que nuestra investigación plantea importantes implicaciones para los hoteleros, clientes y organismos responsables de los destinos, dedicaremos la sección 3 a identificar los efectos de la movilidad para todos estos agentes. En la sección 4 introducimos los conceptos relacionados con la movilidad. En las secciones 5 y 6 , describimos la muestra de hoteles y aplicamos nuestro modelo de análisis de la movilidad a dicha muestra. Por último, en la sección 7 planteamos las conclusiones, así como las limitaciones de nuestra investigación y algunas sugerencias para investigadores interesados en este campo.

\section{MOVILIDAD, RANKINGS Y TRIPADVISOR}

Nuestro trabajo pone por primera vez en contacto tres temas (movilidad, rankings y TripAdvisor), que si bien han sido ampliamente tratados por separado en la literatura científica, apenas se han estudiado de forma conjunta. Por ello revisamos brevemente la bibliografía relacionada con estos tres conceptos.

Con una cierta perspectiva multidisciplinar, desde hace muchos años la movilidad ha sido objeto de incontables estudios en las ciencias sociales. Los economistas, por ejemplo, han analizado la movilidad del factor trabajo (Hunter y Reid, 1968), del factor capital (Sassen, 1990) o la movilidad de los ingresos familiares (Fields, 2000). También ha sido ampliamente estudiada la movilidad desde la sociología, al analizar las diferencias entre el estatus de padres e hijos (Prais, S. 1955; Goodman, 1969), o desde la demografía con innumerables trabajos sobre la movilidad interregional o intraregional (Jeong, 2003).

Pero si hay una ciencia consustancial a la movilidad, ésta es la ciencia turística. El turista es por definición alguien que se mueve y como señala Hall (2005) el turismo es la ciencia social de la movilidad. Es por ello que este concepto ha sido estudiado por investigadores en turismo desde los más variados aspectos: movilidad asociada a los medios de transporte (Löfgren, 2015), relacionada con las personas discapacitadas o con movilidad reducida (Ee Kim y Lehto, 2012), asociada a los flujos turísticos en el tiempo y al análisis de la estacionalidad (Fernández-Morales, 2017), o relacionada con las nuevas tecnologías, conectividad y seguimiento de los movimientos de los turistas en el destino (Shoval y Isaacson, 2009, Ferrante et al., 2018). Como vemos, el concepto de movilidad, si bien con distintos significados, se encuentra presente de forma transversal, en todas las líneas de investigación en turismo. En este trabajo aportamos una aproximación metodológica novedosa que afronta el análisis de la movilidad referida 
no a componentes territoriales o temporales de los individuos o flujos turísticos, sino a los elementos de las ordenaciones o rankings de establecimientos del sector, de manera que se contribuye a la construcción del corpus de conocimiento particular del turismo.

También la literatura científica sobre los rankings es numerosa en el ámbito del turismo, pero lejos de analizar clasificaciones de hoteles o restaurantes, presentan en su práctica totalidad un marcado carácter bibliométrico, centrándose en el estudio de rankings de revistas científicas (Ferreira et al. 1994; Ryan, 2005) o clasificación de autores y citas (Sheldon, 1991). Son también innumerables los trabajos académicos que analizan los famosos y polémicos rankings de universidades (Buela-Casal et al. 2007).

Finalmente, en cuanto a la producción científica centrada en plataformas como TripAdvisor, es necesario recordar brevemente que esta página web ofrece a los investigadores dos fuentes de información: En primer lugar, un repositorio con cientos de miles de comentarios, valoraciones y fotografías aportadas por clientes y turistas, sobre una gran variedad de alojamientos (hoteles, hostales, alquileres vacacionales...), restaurantes y atracciones (museos, monumentos...). Pero, en segundo lugar, aunque no menos importante, TripAdvisor también se caracteriza por ofrecer unos influyentes rankings donde ordena y clasifica todos estos establecimientos en función de aquellas opiniones y valoraciones de los usuarios.

Pues bien, TripAdvisor como repositorio de comentarios ha sido analizado desde innumerables puntos de vista en cientos de trabajos científicos. Sin embargo, llama la atención que TripAdvisor como creador de rankings apenas ha merecido la atención de unos pocos investigadores pese a que es ampliamente reconocida la enorme influencia que estas clasificaciones ejercen en el sector.

Entre la amplia lista de trabajos que analizan la información que TripAdvisor ofrece como repositorio de opiniones y valoraciones encontramos, en primer lugar, aquellos que examinan los comentarios desde la perspectiva de los hoteleros, y estudian entre otros temas, la importancia de los comentarios para el valor de la marca (Callarisa-Fiol et al., 2012), el impacto que las valoraciones de los clientes tiene en la intención de reservar un hotel (Verma et al. 2012), o la relación entre el tamaño del hotel, medido por el número de habitaciones y la valoración global media, (Molinillo et al. 2016). La importancia de conseguir un volumen considerable de comentarios ha sido estudiada por Melián et al. (2010), quienes recomiendan a los directores de hotel que fomenten la participación de sus clientes, ya que, según estos autores, cuantas menos valoraciones recibe un hotel más negativas son las mismas.

Hay también una gran cantidad de investigadores que, desde el punto de vista del usuario, han estudiado, por ejemplo, el proceso de planificación del viaje, la influencia de las opiniones online de otros turistas, así como las características diferenciales entre el usuario y el escritor de comentarios (Gretzel, 2007). En esta línea, ha merecido una atención especial el asunto de las opiniones fraudulentas. Así, Ong (2012) se preguntó si la cantidad de comentarios afecta a la fiabilidad percibida de los mismos, concluyendo que efectivamente un alto número de opiniones proporciona credibilidad. Para Ott et al. (2012), las webs con una baja dificultad para escribir comentarios y ponerlos al alcance de una gran audiencia, tienen un nivel de falsos comentarios mayor que aquellas páginas donde existen más dificultades para publicar una opinión. Incluso llegan a cuantificar el volumen de comentarios fraudulentos señalando que TripAdvisor tiene una tasa aproximada del $4 \%$ de opiniones de este tipo. 
Fruto de esta preocupación por el fraude, en diciembre de 2014 la ICA (Italian Competition Authority) sancionó con una multa de 500.000 Euros a TripAdvisor por infringir varios artículos del código de comercio italiano (Biffaro 2015). El ICA encontró desleal la falta de medidas por parte de TripAdvisor para atajar las opiniones engañosas. No obstante, la mayor medida que tiene TripAdvisor para luchar contra los comentarios fraudulentos es el elevado número de opiniones por hotel. Así, Hensens (2010), en su investigación sobre el fraude en los comentarios refleja que los hoteles con mayor número de opiniones son más insensibles a los falsos comentarios, considerando que a partir de cien opiniones un hotel es prácticamente inmune a los comentarios fraudulentos.

Como indicábamos anteriormente, TripAdvisor es para la mayoría de los investigadores un repositorio de valoraciones, siendo muy pocos los que se interesan por analizar de esta página web lo que para nosotros es la clave de su éxito y su influencia: el diseño y gestión de rankings de hoteles y restaurantes.

Queremos destacar a Smyth et al. (2010) como uno de los primeros trabajos que estudia la evolución en el tiempo del ranking de TripAdvisor, en este caso comparando la variación temporal de las puntuaciones en una muestra de hoteles irlandeses con otra de hoteles de Las Vegas, descubriendo que mientras la puntuación media de los hoteles norteamericanos se había mantenido constante en torno a 3,8 puntos, los hoteles irlandeses pasaron en los mismos dos años de una puntuación media de 3,6 a 3,8. Para los autores esto es debido a lo que ellos llaman "el efecto TripAdvisor". Mientras los hoteles de Las Vegas hace ya tiempo que alcanzaron unos aceptables niveles de calidad, los hoteleros irlandeses, siendo conscientes de que unos comentarios negativos en TripAdvisor podían dañar la imagen de su establecimiento, trataron de mejorar sus niveles de servicio para evitar futuros comentarios negativos.

También Jurca et al. (2010), aunque desde otra perspectiva, estudian la evolución a lo largo del tiempo de las posiciones de los hoteles en el ranking. Analizan un hotel concreto de Nueva York, y conforme van apareciendo nuevos comentarios, los resultados muestran que la mediana y la moda de las puntuaciones proporcionan unos rankings mucho más estables que la media aritmética.

Para Scott y Orlikowski (2012) aunque los mecanismos de rankings no son nuevos, han adquirido gran capacidad de influencia y poder cuando se ejecutan a través de las tecnologías de la web 2.0, redistribuyendo la responsabilidad. El poder de los rankings está en su capacidad de presentarse como una herramienta objetiva sin sesgo, mostrando la verdad, imponiendo su manera de ver las cosas, pero sin ser visto. Además, TripAdvisor tiene un efecto material en los negocios y en la gestión. De hecho, para ciertas empresas del sector turístico una peor o mejor calificación en el ranking puede suponer la diferencia entre las pérdidas o las ganancias, sobreviviendo una temporada o teniendo que cerrar. De esta manera, TripAdvisor es en la práctica una nueva especie de autoridad que dirige las vidas de quienes se dedican al negocio hotelero.

En definitiva, durante los últimos años han sido numerosas las aportaciones científicas al análisis de TripAdvisor desde distintas perspectivas, lo que constituye una prueba evidente de la gran preocupación que su capacidad para influir en clientes y empresas ha generado entre los investigadores. Pero hay que hacer notar una clara desproporción entre los trabajos que estudian a TripAdvisor como repositorio de comentarios, opiniones y valoraciones, y los que lo hacen como diseñador y gestor de rankings, abundantes los primeros y escasos los segundos. 


\section{MOVILIDAD DE UN RANKING: IMPLICACIONES}

Antes de iniciar nuestro análisis, y para entender su importancia, nos parece necesario presentar unas breves reflexiones sobre los efectos que la mayor o menor movilidad puede tener sobre cada uno de los agentes implicados en la industria hotelera.

En función del grado de movilidad, el ranking de una ciudad se encontrará entre uno de los dos casos extremos que definimos a continuación:

Por una parte, tendremos un "ranking sólido" cuando en una ciudad la clasificación de los hoteles presente un grado de movilidad mínimo. En este caso, los hoteles permanecen prácticamente en sus mismas posiciones a lo largo del tiempo, y por tanto los establecimientos situados en la mejor zona del ranking seguirán con una elevada probabilidad en esa misma zona en los próximos meses o años. Igualmente, los situados en las zonas medias y bajas también permanecerán en esas zonas en el futuro.

En el otro extremo, hablamos de un "ranking líquido", con máxima movilidad, cuando los hoteles fluyen con facilidad entre las zonas alta, media y baja. Aquellos que hace unos meses estaban situados en las mejores posiciones, se encuentran ahora en la zona baja, y los que ocupaban los peores puestos, están ahora situados en la zona alta de la clasificación.

Las implicaciones que la mayor o menor movilidad del ranking puede tener para los distintos agentes implicados son dignas de consideración y presentan una amplia gama de importantes consecuencias tanto positivas como negativas para la industria turística:

i) Para los hoteleros: Ante un ranking sólido, no tendría sentido relacionar la posición en el ranking y su más que improbable mejora con fijación de objetivos, políticas retributivas, incentivos al personal o medidas del desempeño a corto o medio plazo.

Los hoteles situados en la zona alta seguirán, en su mayoría, disfrutando de una privilegiada posición en términos de reputación y visibilidad, mientras que la mayor parte de los hoteles situados en la zona central y baja permanecerán por largo tiempo en esas mismas (o similares) posiciones con mínimas probabilidades de alcanzar los mejores puestos.

Por el contrario, un ranking líquido podría considerarse una gran oportunidad, donde todos, grandes cadenas y pequeños hoteles pueden llegar a ser el número uno de la ciudad en un plazo razonable de tiempo.

Las compañías hoteleras que operan en varias ciudades deberían tener en cuenta el correspondiente índice de movilidad del ranking de cada localidad. Sería un grave error comparar la evolución de las posiciones de sus hoteles en ciudades con índices de movilidad sensiblemente distintos, pues una gestión similar tendrá efectos en la clasificación muy diferentes en función del nivel de movilidad del ranking de cada ciudad.

ii) Para los inversores: También debemos plantear la cuestión de si los inversores, o las propias compañías hoteleras, preferirán invertir en ciudades donde los rankings sean sólidos, o por el contrario les resultarán más atractivas las ciudades con rankings líquidos, donde hay una mayor facilidad para alcanzar los mejores puestos en la clasificación, aunque también para caer a las posiciones más bajas. Para aclarar esta cuestión es necesario distinguir entre apertura de nuevos establecimientos y adquisición de establecimientos ya existentes. 
Si se está planteando la apertura de un nuevo hotel, puede ser una gran oportunidad invertir en ciudades con poca movilidad. Un nuevo establecimiento tiene una gran ventaja con respecto a los ya existentes, pues tiene la posibilidad, precisamente por ser nuevo, de situarse en pocos meses en las mejores posiciones. Si además hay poca movilidad, esto le permitirá una vez alcanzada una buena posición, permanecer en ella por largo tiempo. El hotel Room Mate Valeria en Málaga (España) es un claro ejemplo de esto. Se inauguró a principios de 2016 y en agosto de ese mismo año alcanzó el puesto número uno en la ciudad. Un año después (octubre de 2017) continuaba en esa posición.

Cuando de lo que se trata, no es de abrir un nuevo establecimiento, sino de comprar uno ya existente, los inversores deben ser conscientes de que si adquieren un hotel situado en la zona media o baja del ranking en una ciudad con escasa movilidad, son mínimas las posibilidades de situar su hotel a medio plazo en lo más alto de la clasificación. Por tanto, si el objetivo es llegar a estar entre los mejores, en ciudades con rankings sólidos deberían limitar sus compras a hoteles ya situados en la zona alta (al margen de otras consideraciones como rentabilidad, posicionamiento, estrategia competitiva, etc.).

En este sentido, en el proceso de adquisición de un hotel en ciudades con poca movilidad, se debería aplicar una prima al precio de compraventa si el establecimiento se encuentra situado entre los mejores, pues es muy probable que permanezca en esa misma posición a medio y largo plazo, simplemente por estar en una ciudad con poca o nula movilidad. Y en sentido contrario, habría que aplicar un descuento al precio de compraventa en el caso de que el establecimiento estuviera situado en la zona media o baja, ya que difícilmente abandonará esos puestos.

iii) Para los clientes: Es razonable pensar que cuando un cliente busca un hotel, en igualdad de condiciones de la oferta (precio, disponibilidad, localización, instalaciones, etc.) preferirá un establecimiento situado en la zona alta que uno situado en los últimos puestos. Es obvio, por tanto, que la posición en el ranking ejerce una influencia considerable a la hora de elegir un establecimiento.

Debemos preguntarnos ahora qué sensación experimentará un cliente que tras visitar durante varias semanas el ranking de hoteles de la ciudad elegida para pasar las vacaciones con su familia o sus amigos, observa que todos los hoteles permanecen invariablemente en los mismos puestos. El comprobar que aquellos hoteles de su interés se encuentran siempre en las mismas posiciones le producirá una cierta sensación de seguridad, y le convencerá para decidirse por ese hotel que lleva semanas o meses entre los mejores. Por el contrario, si un usuario observa que el hotel que en principio eligió como el más apropiado para alojarse, y que estaba situado hace unas semanas entre los mejores de la ciudad, se encuentra ahora situado entre los peores, esta variación le generará cierto desasosiego, incertidumbre y desconfianza.

Los rankings sólidos, transmiten al cliente una sensación de seguridad que le facilita su decisión. Por el contrario, los rankings líquidos pueden generar gran desconcierto entre los usuarios haciendo más difícil el proceso de elección del establecimiento.

iv) Para las instituciones públicas: La movilidad del ranking también tiene importantes implicaciones para el destino turístico. Una ciudad donde los hoteles apenas varían 
su posición nos puede indicar una planta hotelera con poca competitividad y donde hay una gran desigualdad entre hoteles (unos muy bien valorados siempre arriba y otros muy mal valorados siempre abajo). Por el contrario, mucha movilidad nos puede indicar un destino muy dinámico, con una oferta competitiva e innovadora y con niveles de calidad muy similares donde un mínimo cambio en la valoración de los clientes hace que un hotel pase de estar en lo más alto a estar en la zona baja de la clasificación. De este modo, el concepto de movilidad en los rankings estaría muy relacionado con la desigualdad (en términos de calidad) de la planta hotelera.

v) Para los creadores de rankings: Por último, las compañías tecnológicas que crean estos rankings (TripAdvisor y Booking principalmente) y que tanta influencia ejercen en consumidores y hoteleros también se pueden ver afectadas por la mayor o menor movilidad de las clasificaciones que ellas mismas diseñan.

Por ejemplo, en casos de rankings sólidos, los usuarios se pueden preguntar para qué escribir comentarios si sus valoraciones no van a afectar ni positiva ni negativamente al puesto que ocupa el hotel, y los hoteleros pueden dejar de solicitar a sus clientes que comenten sus experiencias en estas páginas si saben que no servirán para mejorar su posición. Un ranking sólido, donde encontramos los mismos hoteles en las mismas posiciones mes tras mes, año tras año, (el Hotel Orfila era el $\mathrm{N}^{\circ} 1$ en Madrid en septiembre de 2015, y lo seguía siendo en mayo de 2017, en una ciudad con más de 400 hoteles en el ranking TripAdvisor) puede provocar en el usuario una más que razonable sensación de aburrimiento, y pérdida de interés para seguir visitando una página web con un ranking que apenas cambia. Todo esto podría afectar negativamente al tráfico que reciben. Sin embargo, un ranking líquido, muy dinámico, podría generar más visitas cuando se sabe que la clasificación cambia con relativa frecuencia. Así, para los usuarios podría resultar muy interesante visitar la web para descubrir el nuevo número uno de cada mes, generándose un tráfico adicional de potenciales clientes atraídos por conocer las últimas novedades en el ranking.

Los rankings líquidos, donde los hoteles fluyen entre las posiciones altas, medias y bajas con mucha facilidad, estarían asociados como se indicó anteriormente con una planta hotelera homogénea, con un alto grado de igualdad en la calidad de los establecimientos y por tanto con valoraciones muy similares. Podríamos encontrarnos ante una nueva forma de consultar los rankings, pues el hecho de estar en la zona alta, media, o baja ya no sería tan determinante para elegir un hotel, porque en este caso los clientes más que un ranking, estarían consultando un listado de hoteles donde todos tienen una calidad similar y no habría diferencias significativas entre los de arriba y los de abajo. En cualquier caso, serán estas empresas tecnológicas las que decidirán mediante el diseño de sus algoritmos de clasificación, si el ranking debe tener más o menos movilidad.

En definitiva, la movilidad de los rankings existe, aunque nadie la haya medido todavía. Nosotros lo haremos por primera vez, pues parece evidente que puede ejercer una gran influencia sobre hoteleros, clientes y destinos. Lo que no debemos hacer es juzgarla, esto es, considerarla como una característica positiva o negativa en sí misma. Porque siempre habrá grupos interesados en que el ranking de hoteles o restaurantes 
de su ciudad sea sólido, inamovible. Pero sin duda también encontraremos en la misma ciudad colectivos interesados en que el ranking sea líquido. Por eso hemos querido ilustrar con los párrafos anteriores la complejidad de este concepto y los innumerables pros y contras que ofrece a cada uno de los agentes implicados. La movilidad será al mismo tiempo positiva para unos y negativa para otros.

\section{METODOLOGÍA}

Resulta conveniente, para centrar nuestro estudio, comenzar definiendo algunos conceptos básicos. Definimos en primer lugar un ranking como un listado ordenado de elementos según un criterio previamente establecido. A partir de aquí, para definir la movilidad de un ranking, es necesario distinguir entre macro y micromovilidad.

\subsection{Macro y micromovilidad}

Desde una perspectiva macro, puede definirse la movilidad como la medida de los cambios producidos en las posiciones que ocupan los elementos de un ranking durante un periodo de tiempo. Esta movilidad vendrá determinada por tres factores que hemos dado en llamar las tres C's de la movilidad, por sus iniciales en español: C1-Cuantos, C2-Cuanto y C3-Cuando:

C1 La movilidad de un ranking vendrá determinada por el número de elementos que modifiquen su posición en la clasificación (Cuantos). Si la mayoría de hoteles cambia su posición, habrá más movilidad que si sólo lo hace una pequeña parte de ellos, permaneciendo el resto en el mismo puesto.

C2 La movilidad de un ranking vendrá también determinada por la amplitud de esos cambios (Cuanto). Si los hoteles apenas varían unos pocos puestos habrá menos movilidad que si se producen grandes saltos.

C3 La movilidad de un ranking vendrá por último determinada por la frecuencia con que se produzcan los cambios (Cuando). Si los cambios en la clasificación se producen diariamente habrá más movilidad que si los cambios tienen lugar de forma mensual o trimestral, o simplemente de un modo esporádico.

Hablaremos por tanto de un ranking con alta movilidad, para un determinado periodo de tiempo, cuando experimenten cambios en la clasificación la mayoría de los elementos que lo conforman $(\mathrm{C} 1)$, estos cambios sean significativos (C2) y además sean frecuentes (C3). Hay, por el contrario, escasa movilidad si son pocos los elementos que varíen su posición, si los cambios son irrelevantes o si son infrecuentes.

Un análisis de la macromovilidad es, por tanto, aquel que estudia la movilidad del ranking en su conjunto. Podemos entonces crear un índice de macromovilidad para obtener una valoración global de la movilidad del ranking, esto es, una medida del grado en que las posiciones varían a lo largo del tiempo. Este índice nos permitirá comparar las clasificaciones de distintas ciudades, en distintos momentos del tiempo, o de distintos sectores. Así, por ejemplo, podremos conocer si hay más movilidad en los hoteles de 
Madrid que en los de Barcelona, saber cuánto ha variado la movilidad en el ranking de hoteles de Valencia en los últimos dos años, o comprobar si en Málaga hay más movilidad en los rankings de restaurantes o de hoteles.

Desde una perspectiva micro, definimos la movilidad como la medida de los cambios producidos en las posiciones que ha ocupado un determinado establecimiento en un periodo de tiempo. El análisis de la micromovilidad se encarga por tanto de estudiar el comportamiento de cada uno de los componentes del ranking, analizando las trayectorias individuales a lo largo del tiempo. En este caso, obtendremos un índice de micromovilidad individual, lo que nos permitirá ordenar los hoteles o restaurantes por su grado de movilidad, y realizar comparaciones entre distintos establecimientos para conocer si el Hotel X tiene mayor movilidad que el Hotel Y, o si en los últimos años la movilidad del Restaurante $\mathrm{Z}$ ha aumentado o disminuido.

Aunque las técnicas de análisis macro y micro pueden ser muy diferentes, obviamente las dos formas de estudiar la movilidad están conectadas, pues a partir de un análisis micro, podemos utilizar los valores individuales para de forma agregada realizar un análisis macro. Esta es precisamente la técnica que aplicaremos en este trabajo, donde primero se analizan los hoteles de forma individual para posteriormente estudiarlos de forma conjunta.

Por último y como aclaración terminológica, a lo largo de este artículo utilizaremos principalmente el término movilidad, sabiendo que si hablamos de un ranking, nos estaremos refiriendo implícitamente a la macromovilidad, mientras que si hablamos de los hoteles de forma individual estaremos haciendo referencia a la micromovilidad.

\subsection{Análisis de trayectorias}

Desde un punto de vista metodológico, el análisis de la movilidad requiere en primer lugar que de forma periódica y prolongada en el tiempo se recoja información sobre las posiciones que ocupan en cada momento los elementos que conforman el ranking.

Definimos la trayectoria individual de un establecimiento como el conjunto ordenado de las distintas posiciones que ha ocupado en la clasificación a lo largo de un periodo de tiempo. Podemos considerar la observación y el análisis de estas trayectorias como el objetivo fundamental en el estudio de la movilidad de los rankings. Cada trayectoria individual podrá quedar entonces representada matemáticamente a través de un vector que llamaremos vector trayectoria y que describe, para cada elemento del ranking, las diferentes posiciones que ha ocupado a lo largo del tiempo. Antes de iniciar cualquier estudio de movilidad es necesario, por tanto, configurar el vector trayectoria, lo que supone fijar los tres parámetros siguientes:

1) Dimensión de la trayectoria ( $\mathrm{T}$ ): La definimos como el número de observaciones que se incluyen en el estudio para cada hotel o elemento.

2) Periodicidad del estudio (P): Habrá que determinar si las observaciones se realizarán con periodicidad diaria, semanal, mensual, trimestral, etc. 
3) Número de zonas (cuantiles) en que se va a dividir el ranking (Q): Debemos establecer si el ranking se divide en deciles $(Q=10)$, cuartiles $(Q=4)$, percentiles $(\mathrm{Q}=100)$, etc. o, si por el contrario, por tratarse de rankings pequeños o cuando se requiera una precisión máxima, no se divide este en zonas, sino que se indica la posición exacta que ocupa cada elemento en la clasificación. En este caso, Q = $\mathrm{N}$, siendo $\mathrm{N}$ el número total de hoteles o elementos incluidos en el ranking.

La evolución temporal de cada elemento que forma parte de un ranking, queda representada mediante un vector trayectoria $(\mathbf{m})$ de dimensión $\mathrm{T}$, donde cada uno de los elementos $\left(\mathrm{m}_{\mathrm{i}}\right)$ que componen el vector podrá tomar valores entre 1 y $\mathrm{Q}$.

$$
\mathbf{m}=\left(\begin{array}{llll}
m_{1} & m_{2} & m_{3} & \ldots \\
\mathrm{m}
\end{array}\right)
$$

Debemos aclarar, que si bien es frecuente en estudios estadísticos la identificación de los cuantiles más elevados con los valores más "favorables" o "excelentes" (por ejemplo, en estudios de rentas o salarios, los deciles 1 y 2 se corresponden con las rentas y salarios menores, en tanto que el decil 10 corresponde a los mayores ingresos y salarios); en nuestro caso, y dado que en un ranking de hoteles o restaurantes el puesto número 1 se asocia al mejor establecimiento y la posición en el ranking varía inversamente con el grado de excelencia revelado por el usuario, calificaremos como las zonas más elevadas del ranking las correspondientes a los valores más bajos de la posición ocupada, y del mismo modo, cuando trabajamos con zonas del ranking y cuantiles, el decil 1 corresponde a la zona más alta, y el decil 10 a la zona más baja del ranking.

Así por ejemplo, y para un ranking con $\mathrm{Q}=10$, el vector trayectoria $\mathbf{m}=(6,7,5,5$, 6) indica que en el primer periodo analizado el hotel estaba situado en el decil 6, en el segundo periodo bajó al decil 7, en el tercero subió al decil 5, en el cuarto continuó en el decil 5 y finalmente bajó al decil 6.

\subsection{Indicadores parciales de micromovilidad}

A partir de los datos recogidos en el vector trayectoria podemos definir una serie de variables que nos ayudarán a conocer el comportamiento de un hotel a lo largo del tiempo, y en última instancia medir su grado de movilidad, lo que nos permitirá clasificarlo y compararlo con otros hoteles. Aunque estas variables serán utilizadas para crear índices de micro y de macromovilidad, nos ofrecen por sí mismas suficiente información para encontrar evidencias sobre la mayor o menor movilidad de un hotel (analizadas de forma individual) o de un ranking (si se analizan de forma agregada). Las variables que hemos definido y que podremos obtener a partir del vector trayectoria están relacionadas con el número de zonas del ranking en las que el hotel o restaurante ha estado ( $\left.\mathrm{IP}_{1}\right)$, el número de rachas observadas en su trayectoria $\left(\mathrm{IP}_{2}\right)$ y la amplitud o distancia de los cambios experimentados en las posiciones del ranking $\left(\mathrm{IP}_{3}, \mathrm{IP}_{4}, \mathrm{IP}_{5}\right)$.

$\mathrm{IP}_{1}$ : Definimos este indicador como el número de zonas distintas del ranking (cuantiles) en las que se ha situado un hotel a lo largo de un determinado periodo de tiempo. Podemos considerar esta variable como un primer indicador parcial del grado de movilidad de 
un establecimiento. Así un hotel que durante todo el periodo de estudio ha permanecido siempre en la misma zona del ranking $\left(\mathrm{IP}_{1}=1\right)$ se considerará un hotel con nula movilidad, mientras que un hotel que ha pasado por distintas zonas será considerado un hotel con cierto grado de movilidad. Tendrá por tanto un mayor grado de movilidad aquel hotel que visite un mayor número de zonas distintas. Esta variable oscila entre un nivel mínimo $\mathrm{IP}_{1}$ = 1 y un nivel máximo de $\mathrm{IP}_{1}=$ mín $(\mathrm{T}, \mathrm{Q})$ donde $\mathrm{T}$ es el número de periodos analizados, y $\mathrm{Q}$ el número de zonas o cuantiles en que se divide el ranking.

El número de zonas del ranking que ha visitado un hotel en su trayectoria no es suficiente para conocer su nivel de movilidad. Así, por ejemplo, aunque estas dos trayectorias, $(1,1,1,1,2)$ y $(1,2,1,2,1)$, contienen el mismo número de zonas distintas $\left(\operatorname{IP}_{1}=2\right)$, son muy diferentes en términos de movilidad. El hotel correspondiente al primer vector trayectoria ha permanecido prácticamente todo el tiempo en la misma zona, mientras que el segundo ha estado saltando continuamente de un cuantil a otro. Podemos recurrir al concepto de rachas, ampliamente utilizado en estadística (Salamanca et al., 2017; Babativa y Corzo, 2010; O’Brien y Dyck, 1985; Nicholson, 1986; Fu y Koutras, 1994; Driscoll et al., 1994) para complementar nuestro análisis, mediante el segundo indicador parcial de movilidad.

$\mathrm{IP}_{2}$ : Definimos este indicador como el número de rachas presente en el vector trayectoria, entendiendo las rachas como el conjunto de periodos consecutivos en los que la posición del hotel permanece invariable. Así, en el primer ejemplo encontramos dos rachas, y en el segundo cinco. Cuantas más rachas tenga una trayectoria, mayor será la movilidad. El valor mínimo de esta variable es $\mathrm{IP}_{2}=1$, siendo el valor máximo $\mathrm{IP}_{2}=\mathrm{T}$.

El tercer elemento que consideramos para la elaboración de índices parciales de movilidad es la distancia o amplitud de los cambios o saltos en el ranking que experimenta cada elemento.

Entre toda la información que nos ofrece el análisis de las trayectorias individuales de los hoteles, quizás la variable más asociada al concepto de movilidad sea la distancia recorrida por el hotel entre su posición inicial y final, ya que, de forma intuitiva, cuando queremos analizar cómo ha evolucionado un hotel a lo largo del tiempo lo primero que hacemos es comparar su posición al inicio y al final del periodo. El indicador $\mathrm{IP}_{3}$ nos ayuda a cuantificar esta información.

$\mathrm{IP}_{3}$ : Definimos este indicador como la diferencia entre el primer y el último elemento del vector trayectoria. Así, diremos que un hotel con una trayectoria $(6,6,5,5,4)$ ha mejorado su posición dos deciles a lo largo del periodo $\left(\mathrm{IP}_{3}=2\right)$, mientras que el hotel con trayectoria $(3,4,5,5,6)$ ha empeorado su situación en el ranking entre el momento inicial y final en tres deciles $\left(\mathrm{IP}_{3}=-3\right)$. Esta variable toma valores positivos y negativos, indicando los primeros una mejora en la clasificación y los segundos un empeoramiento en la posición en el ranking. El indicador $\mathrm{IP}_{3}$ oscila entre un valor mínimo de $\mathrm{IP}_{3}=1-\mathrm{Q}$ (en nuestro ejemplo $Q=10$ ), que corresponde con una caída máxima en la clasificación pasando del primer al último cuantil; y el valor máximo $\mathrm{IP}_{3}=\mathrm{Q}-1$, que indica una subida desde la zona más baja a la zona más alta del ranking.

Aunque la distancia entre la posición inicial y final aporta gran información para determinar la movilidad de un hotel a lo largo del tiempo, es conveniente completar el análisis con la información que proporciona la distancia entre el cuantil máximo y mínimo 
visitado, con independencia del momento en el que se haya hecho. Así, por ejemplo, la trayectoria $(3,4,5,4,3)$, aunque no presenta cambios entre la posición inicial y final $\left(\mathrm{IP}_{3}\right.$ $=0$ ), sí ha experimentado, por el contrario, un cambio de dos deciles a lo largo del periodo entre el máximo y el mínimo, lo que nos muestra cierto grado de movilidad, que el indicador parcial de movilidad $\mathrm{IP}_{3}$ no refleja. Por este motivo, construimos el indicador $\mathrm{IP}_{4}$.

$\mathrm{IP}_{4}$ : Definimos este indicador como la diferencia entre el mayor y el menor elemento del vector trayectoria. Por tanto, cuantifica la distancia entre el máximo y el mínimo cuantil por donde el establecimiento ha pasado, con independencia del momento en el que se hayan alcanzado dichas posiciones. Esta variable tiene un valor mínimo $\mathrm{IP}_{4}=0$ cuando no hay diferencias entre la posición máxima y mínima y un valor máximo $\mathrm{IP}_{4}=\mathrm{Q}-1$.

Por último, es posible complementar el conjunto de indicadores con una medida de las distancias recorridas en dos periodos consecutivos. En este caso, al existir varias distancias (tantas como periodos consecutivos), debemos recurrir a alguna función que identifique esta variable. Así, se puede utilizar el máximo de las distancias entre dos periodos consecutivos, el mínimo, el promedio, la desviación típica, la suma, etc., tanto en valores reales como absolutos. Podemos proponer como más adecuada la función máximo para analizar la distancia entre periodos para definir el indicador $\mathrm{IP}_{5}$.

$\mathrm{IP}_{5}$ : Definimos este indicador como el valor máximo de las diferencias entre cada dos elementos consecutivos del vector trayectoria. Por lo tanto, este indicador cuantifica el mayor salto en su posición que ha dado el hotel entre momentos consecutivos.

Como vemos, todos estos indicadores ofrecen información diferente y útil para conocer la movilidad de un hotel a través de su vector trayectoria. Por limitaciones de espacio, en el presente trabajo utilizaremos los cuatro primeros indicadores, dejando el estudio de las distancias de periodos consecutivos, por su considerable extensión, para futuros trabajos.

\section{MUESTRA Y BASE DE DATOS}

Este trabajo forma parte de un proyecto de investigación más amplio que tiene por objetivo estudiar el funcionamiento y la evolución de los rankings de hoteles y restaurantes en las principales ciudades del mundo, tanto en TripAdvisor como en Booking. Para ello, desde de junio de 2015, con periodicidad semanal, se han extraído de forma ininterrumpida las posiciones que los distintos hoteles ocupan en los rankings de sus respectivas ciudades.

En este artículo se analiza la movilidad de los rankings de TripAdvisor de las seis primeras capitales de provincia españolas por número de habitantes (a 1 de enero de 2015), que son a su vez las únicas que superan la barrera de los 500.000 habitantes. El cuadro 1 presenta la distribución de los hoteles de la muestra por ciudades, así como el porcentaje que representan respecto del número total de hoteles presentes en TripAdvisor (en ningún caso inferior al $48 \%$ ).

Debido a que la movilidad detectada, tanto semanal como mensual, es de escasa magnitud, se ha decidido usar los datos trimestrales, donde ya se puede apreciar ciertos niveles de movilidad. Por tanto, el presente trabajo analiza la evolución trimestral a lo largo de un año (de junio de 2015 a junio de 2016) de los rankings de hoteles de las seis ciudades mencionadas. De este modo, para el periodo analizado hemos obtenido cinco posiciones 
en el ranking para cada hotel (junio, septiembre y diciembre de 2015 y marzo y junio de 2016). Una vez procesada esta información, se han obtenido los vectores trayectoria para cada establecimiento, reflejando la evolución de sus posiciones a lo largo del periodo.

Asimismo, debemos señalar que los rankings han sido divididos en deciles $(Q=10)$, y que como se indicó anteriormente $\mathrm{Q}=1$ corresponde a la zona más alta del ranking, donde se encuentran las mejores posiciones, mientras que $\mathrm{Q}=10$, se refiere a la zona más baja del ranking donde se encuentran los establecimientos peor clasificados.

\section{Cuadro 1 \\ DISTRIBUCIÓN DE LA MUESTRA}

\begin{tabular}{lccccc}
\hline Ciudad & $\begin{array}{c}\text { Hoteles en } \\
\text { la muestra }\end{array}$ & $\begin{array}{c}\text { Población a } \\
01 / 01 / 2015^{1}\end{array}$ & $\begin{array}{c}\text { Distribución } \\
\text { por ciudades } \\
\text { de la muestra (\%) }\end{array}$ & $\begin{array}{c}\text { Hoteles en } \\
\text { TripAdvisor }{ }^{2}\end{array}$ & $\begin{array}{c}\text { Relación hoteles } \\
\text { en la muestra y en } \\
\text { TripAdvisor (\%) }\end{array}$ \\
\hline Barcelona & 271 & 1.604 .555 & 35,15 & 524 & 51,72 \\
Madrid & 250 & 3.141 .991 & 32,43 & 447 & 55,93 \\
Sevilla & 104 & 693.878 & 13,49 & 176 & 59,09 \\
Valencia & 55 & 786.189 & 7,13 & 100 & 55,00 \\
Zaragoza & 47 & 664.953 & 6,10 & 80 & 58,75 \\
Málaga & 44 & 569.130 & 5,71 & 90 & 48,89 \\
\hline Total & 771 & & 100,00 & 1.417 & 54,41 \\
\hline
\end{tabular}

Fuente: Elaboración propia a partir de los datos extraídos de TripAdvisor ${ }^{3}$.

Tras seleccionar el total de hoteles recogidos en el ranking de Tripadvisor, se decidió eliminar de la muestra aquellos establecimientos que no tuvieran ningún comentario ni en el momento inicial ni en ninguno de los periodos posteriores. Son pequeños establecimientos casi marginales sobre los que nadie ha opinado. Al no tener valoraciones de clientes tampoco tienen una puntuación global media, por lo que TripAdvisor los sitúa al final del ranking pero sin asignarles una posición numérica.

En segundo lugar se han suprimido los hoteles con datos faltantes en algún periodo, por lo que la muestra final está compuesta de todos aquellos hoteles para los que se dispone de información en todos y cada uno de los momentos en que se tomaron datos durante la investigación.

Al ser un análisis longitudinal, los autores se han encontrado como es habitual en este tipo de estudios, con elementos ausentes en alguno de los periodos. La pérdida o no disponibilidad de una proporción variable de los datos correspondientes a los sujetos seleccionados es siempre una realidad a considerar en todo estudio de investigación (Durán, 2005) y uno de los problemas metodológicos en estudios longitudinales (Twisk y De Vente, 2001)

\footnotetext{
1 Fuente: Instituto Nacional de Estadística. (01/01/2015).

2 Número de Hoteles en TripAdvisor a 30/06/2015.

3 Todos los cuadros y gráficos que aparecen en este artículo han sido elaborados por los autores a partir de los datos exrtraídos de TripAdvisor.
} 
Por ello, identificar los determinantes de la pérdida de información es relevante para una correcta interpretación de los resultados (Twisk y De Vente, 2001). Así, en relación a nuestro trabajo, las razones por las que un hotel puede no aparecer en alguno de los momentos en que se tomaron los datos son básicamente cuatro:

1) Nacimiento de nuevos hoteles: El hotel no existía al inicio de nuestra investigación (junio 2015), y se inauguró en algún momento a lo largo del periodo de estudio, por lo que tendríamos datos faltantes en los primeros momentos.

2) Mortalidad: Hoteles que estaban en funcionamiento en junio de 2015 pero han cerrado durante los meses en que se realizó el estudio, por lo que nos encontraríamos con datos faltantes en los últimos periodos.

3) Incidencias técnicas: Al realizar el proceso de extracción de datos de forma automatizada cualquier mínima incidencia técnica en ordenadores, servidores, conexión a Internet, o cambios en el diseño de la página web de TripAdvisor puede provocar la existencia de datos faltantes.

4) Por último, nunca se pueden descartar errores humanos en el procesamiento y manipulación de la información.

Los datos perdidos durante una investigación pueden causar principalmente dos tipos de problemas: En primer lugar pérdida de potencia o precisión en los resultados debido a la reducción del número de casos analizados. En segundo lugar pueden afectar a la validez de los resultados debido a posibles sesgos (Deeg, 2000; Durán 2005). En nuestro caso, y aunque se podrían haber aplicado diferentes técnicas que permiten reemplazar matemáticamente los datos perdidos (Engels y Diehr, 2003), no se ha considerado necesario ya que la muestra final (771 hoteles con datos en todos los periodos) es suficientemente representativa por lo que ni la precisión ni la validez de los resultados se ven afectadas de forma significativa por los datos faltantes.

\section{ANÁLISIS DE LOS RESULTADOS}

En esta sección analizamos los resultados obtenidos de la estimación de los vectores trayectoria y los cuatro indicadores de movilidad, $\mathrm{IP}_{1}$ a $\mathrm{IP}_{4}$, observados en nuestra muestra de 771 hoteles españoles.

\subsection{Variabilidad en las trayectorias}

Para realizar el análisis de las distintas zonas del ranking que ha visitado cada hotel, presentamos en primer lugar, en el cuadro 2, la distribución de los hoteles de la muestra según el decil que ocupaban en el momento de iniciarse el estudio (Jun-15) y por columnas según el indicador $\mathrm{IP}_{1}$ (número de deciles distintos por los que han pasado esos hoteles a lo largo del periodo analizado). En este sentido, para facilitar el análisis, clasificamos los establecimientos según este indicador distinguiendo entre de hoteles con nula $\left(\operatorname{IP}_{1}=1\right)$, poca $\left(\operatorname{IP}_{1}=2\right)$, media $\left(\operatorname{IP}_{1}=3\right)$, alta $\left(\operatorname{IP}_{1}=4\right)$ y máxima $\left(\operatorname{IP}_{1}=5\right)$ variabilidad. 


\section{Cuadro 2 \\ DISTRIBUCIÓN DE HOTELES SEGÚN IP 1 Y DECIL INICIAL (\%)}

\begin{tabular}{ccccccc}
\hline \multicolumn{7}{c}{ IP $1_{1}$} \\
\hline Decil Inicial & 1 & 2 & 3 & 4 & 5 & Total \\
\hline 1 & 79,00 & 20,00 & 1,00 & 0,00 & 0,00 & 100 \\
2 & 41,57 & 46,07 & 10,11 & 2,25 & 0,00 & 100 \\
3 & 32,53 & 53,01 & 13,25 & 0,00 & 1,20 & 100 \\
4 & 15,38 & 58,24 & 23,08 & 3,30 & 0,00 & 100 \\
5 & 15,48 & 64,29 & 17,86 & 2,38 & 0,00 & 100 \\
6 & 11,54 & 65,38 & 19,23 & 2,56 & 1,28 & 100 \\
7 & 25,64 & 61,54 & 12,82 & 0,00 & 0,00 & 100 \\
8 & 26,74 & 60,47 & 10,47 & 1,16 & 1,16 & 100 \\
9 & 33,90 & 62,71 & 3,39 & 0,00 & 0,00 & 100 \\
10 & 60,87 & 30,43 & 8,70 & 0,00 & 0,00 & 100 \\
\hline Total & 33,20 & 52,79 & 12,32 & 1,30 & 0,39 & 100 \\
\hline
\end{tabular}

Entre los distintos resultados que sobre la movilidad de los hoteles nos ofrece el cuadro 2, destaca en primer lugar el hecho de que del total de hoteles que en Jun-2015 estaban en la zona más alta del ranking (Decil 1), el 79\% no ha dejado esa zona durante todo el año. En general, son mayoría los hoteles que han visitado dos zonas del ranking $(52,79 \%)$, siendo también muy considerable el número de establecimientos de la muestra que ha permanecido en la misma zona $(33,20 \%)$. Por tanto, el $85,99 \%$ ha estado como máximo en dos deciles distintos en todo el periodo, lo que correspondería a establecimientos con poca o nula movilidad. Sólo hay un 0,39\% de hoteles que ha visitado cinco deciles distintos en las cinco fechas estudiadas, siendo $1,30 \%$ el porcentaje de hoteles que ha estado en cuatro deciles distintos. Por tanto, es casi insignificante $(1,69 \%)$ la cifra de hoteles que han visitado cuatro o cinco deciles distintos y que corresponderían a los hoteles con un alto grado de movilidad.

Si representamos gráficamente sólo la primera columna del cuadro 2 (los hoteles que han permanecido siempre en el mismo decil) observamos cierta relación entre estos porcentajes y la posición que ocupaba el hotel en la clasificación al inicio del periodo (Figura 1). Así, los hoteles situados en la zona alta (decil 1) y baja (decil 10) tienden a permanecer en esa misma zona en mayor proporción que los hoteles situados en las zonas centrales.

Hay, por tanto, ciertas evidencias de lo que podríamos llamar Efecto Primer/Último Decil, indicando que la movilidad en los extremos es muy inferior a la que se produce en las zonas centrales del ranking. Se podrían considerar a los deciles 1 y 10 como zonas con un alto grado de absorción porque cuando un hotel entra en una de estas zonas tiene una elevada probabilidad de permanecer en ella durante largo tiempo.

Por último, en promedio, los hoteles han visitado 1,82 deciles durante el periodo de estudio, cifra que nos muestra un nivel de movilidad muy bajo. La desviación estándar se sitúa en 0,71 lo que nos indica un grado de concentración en torno a la media suficiente para considerar al promedio como representativo. En definitiva, el análisis de esta variable nos muestra un porcentaje muy elevado de hoteles con poca o nula movilidad $(85,99 \%)$, mientras que son muy escasos los establecimientos con movilidad alta o máxima $(1,69 \%)$. 


\section{Figura 1}

HOTELES QUE NO CAMBIAN DE DECIL (\%)

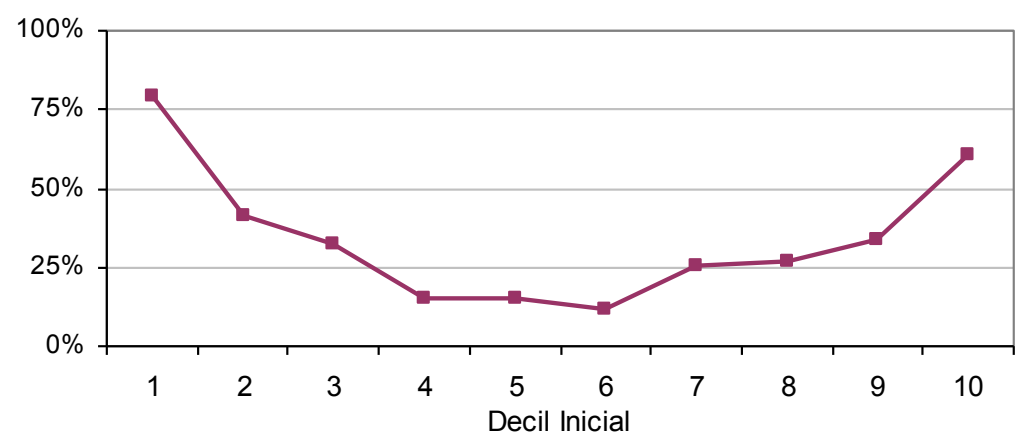

\subsection{Rachas en las trayectorias observadas en la muestra}

El cuadro 3 presenta los hoteles de la muestra agrupados en columnas por el indicador $\mathrm{IP}_{2}$ (número de rachas en sus respectivas trayectorias). Las filas siguen agrupando los hoteles por el decil en el que se encontraban situados en el momento inicial.

\section{Cuadro 3}

DISTRIBUCIÓN DE HOTELES SEGÚN IP 2 Y DECIL INICIAL (\%)

\begin{tabular}{ccccccc}
\hline \multicolumn{7}{c}{$\mathrm{IP}_{2}$} \\
\hline Decil Inicial & 1 & 2 & 3 & 4 & 5 & Total \\
\hline 1 & 79,00 & 15,00 & 3,00 & 3,00 & 0,00 & 100 \\
2 & 41,57 & 31,46 & 17,98 & 6,74 & 2,25 & 100 \\
3 & 32,53 & 34,94 & 26,51 & 3,61 & 2,41 & 100 \\
4 & 15,38 & 31,87 & 39,56 & 10,99 & 2,20 & 100 \\
5 & 15,48 & 35,71 & 28,57 & 16,67 & 3,57 & 100 \\
6 & 11,54 & 30,77 & 34,62 & 15,38 & 7,69 & 100 \\
7 & 25,64 & 44,87 & 21,79 & 5,13 & 2,56 & 100 \\
8 & 26,74 & 44,19 & 16,28 & 10,47 & 2,33 & 100 \\
9 & 33,90 & 49,15 & 13,56 & 3,39 & 0,00 & 100 \\
10 & 60,87 & 13,04 & 17,39 & 8,70 & 0,00 & 100 \\
\hline Total & 33,20 & 33,72 & 22,18 & 8,43 & 2,46 & 100 \\
\hline
\end{tabular}

Observamos que la primera columna del cuadro 3 coincide con la primera columna del cuadro 2, debido a que cuando $\mathrm{IP}_{1}=1$ entonces $\mathrm{IP}_{2}=1$. Esta coincidencia sólo ocurre necesariamente cuando $\mathrm{IP}_{1}=1$. Así, mientras los hoteles con $\mathrm{IP}_{1}=2$, esto es, hoteles que han visitado dos zonas del ranking, representan el 52,79\% del total de la muestra, los hoteles con dos rachas en su trayectoria sólo representan el 33,72\%. 
En general, se observa que los hoteles presentan en promedio 2,13 rachas durante el periodo de estudio, cifra que nos vuelve a mostrar un nivel de movilidad muy bajo. La desviación estándar se sitúa en 1,04 por lo que consideramos al promedio como representativo.

\subsection{Distancias en los vectores trayectoria observados}

Los resultados del primer indicador de distancias que analizamos $\left(\mathrm{IP}_{3}\right)$ se presentan en el cuadro 4, que recoge la distribución porcentual de los hoteles de la muestra según el valor de $\mathrm{IP}_{3}$ en columnas y el decil ocupado al inicio del periodo en filas.

\section{Cuadro 4 \\ DISTRIBUCIÓN DE HOTELES SEGÚN IP3 Y DECIL INICIAL (\%)}

\begin{tabular}{|c|c|c|c|c|c|c|c|c|c|c|c|}
\hline & & & & & & $\mathrm{IP}_{3}$ & & & & & \\
\hline $\begin{array}{c}\text { Decil } \\
\text { Inicial }\end{array}$ & -5 & -4 & -3 & -2 & -1 & 0 & 1 & 2 & 3 & 4 & Total \\
\hline 1 & 2,00 & 0,00 & 0,00 & 1,00 & 16,00 & 81,00 & 0,00 & 0,00 & 0,00 & 0,00 & 100 \\
\hline 2 & 1,12 & 4,49 & 5,62 & 8,99 & 10,11 & 52,81 & 16,85 & 0,00 & 0,00 & 0,00 & 100 \\
\hline 3 & 1,20 & 0,00 & 4,82 & 6,02 & 16,87 & 49,40 & 19,28 & 2,41 & 0,00 & 0,00 & 100 \\
\hline 4 & 0,00 & 0,00 & 6,59 & 8,79 & 17,58 & 38,46 & 25,27 & 3,30 & 0,00 & 0,00 & 100 \\
\hline 5 & 0,00 & 0,00 & 1,19 & 5,95 & 16,67 & 38,10 & 30,95 & 5,95 & 1,19 & 0,00 & 100 \\
\hline 6 & 0,00 & 0,00 & 0,00 & 6,41 & 16,67 & 39,74 & 26,92 & 7,69 & 1,28 & 1,28 & 100 \\
\hline 7 & 0,00 & 0,00 & 0,00 & 1,28 & 25,64 & 44,87 & 19,23 & 7,69 & 1,28 & 0,00 & 100 \\
\hline 8 & 0,00 & 0,00 & 0,00 & 1,16 & 32,56 & 40,70 & 20,93 & 2,33 & 0,00 & 2,33 & 100 \\
\hline 9 & 0,00 & 0,00 & 0,00 & 0,00 & 44,07 & 47,46 & 6,78 & 1,69 & 0,00 & 0,00 & 100 \\
\hline 10 & 0,00 & 0,00 & 0,00 & 0,00 & 0,00 & 73,91 & 13,04 & 8,70 & 4,35 & 0,00 & 100 \\
\hline Total & 0,52 & 0,52 & 2,08 & 4,41 & 20,23 & 49,55 & 18,29 & 3,50 & 0,52 & 0,39 & 100 \\
\hline
\end{tabular}

Entre los resultados de mayor interés que nos ofrece el cuadro 4, debemos destacar en primer lugar que el 49,50\% de los hoteles no ha modificado su posición en el ranking entre el momento inicial y final. Recordemos que tanto los valores estimados de la variable $\mathrm{IP}_{1}$, como los de la variable $\mathrm{IP}_{2}$ nos indican que los hoteles que no han modificado su posición en el ranking a lo largo de todo el periodo suponen el 33,20\% de la muestra, un porcentaje significativamente inferior al anterior. La diferencia entre estas dos cifras $(16,30 \%)$ nos muestra el porcentaje de hoteles que tras realizar algún cambio durante la trayectoria, vuelve a su posición inicial en el último periodo.

Es de destacar que una gran mayoría de establecimientos (el 88,07\%) ha modificado su posición en el ranking entre el momento inicial y final en un decil como máximo, ya sea hacia arriba o hacia abajo, llegando al $95.98 \%$ la cifra de hoteles que ha modificado su posición en el ranking entre el momento inicial y final dos deciles como máximo. Esta 
variable, en sintonía con las anteriores, nos muestra unos niveles de movilidad muy bajos. También se observa que es sensiblemente mayor el número de hoteles que ha bajado algún decil en el ranking $(27,76 \%)$ de aquellos que han subido entre el momento inicial y final $(22,70 \%)$. Como ocurría con los indicadores $\mathrm{IP}_{1}$ y $\mathrm{IP}_{2}$, se aprecia con nitidez cómo aumenta la movilidad a medida que nos acercamos al centro desde cualquiera de los dos extremos del ranking. De nuevo encontramos ciertas evidencias de que las zonas extremas son las que presentan un mayor número de hoteles con poca o nula movilidad, en este caso de forma muy destacada la zona alta del ranking donde observamos que el $81,00 \%$ de los hoteles que estaban en esta zona en junio de 2015, siguen ahí un año después.

Por último, el valor promedio de esta variable en valor absoluto es ciertamente bajo $(0,683)$, evidenciando unos niveles de movilidad muy bajos. La desviación estándar $(0,86)$ nos permite considerar al promedio suficientemente representativo.

Analizamos, a continuación, el último indicador de micromovilidad, IP 4. Así, el cuadro 5 nos muestra los valores observados de la distancia máximo-mínimo en la muestra, en relación al decil ocupado al inicio del periodo:

\section{Cuadro 5 \\ DISTRIBUCIÓN DE HOTELES SEGÚN IP4 Y DECIL INICIAL (\%)}

\begin{tabular}{cccccccc}
\hline \multicolumn{7}{c}{$\mathrm{IP}_{4}$} \\
\hline Decil Inicial & 0 & 1 & 2 & 3 & 4 & 5 & Total \\
\hline 1 & 79,00 & 18,00 & 1,00 & 0,00 & 0,00 & 2,00 & 100 \\
2 & 41,57 & 38,20 & 8,99 & 4,49 & 5,62 & 1,12 & 100 \\
3 & 32,53 & 50,60 & 9,64 & 6,02 & 0,00 & 1,20 & 100 \\
4 & 15,38 & 53,85 & 20,88 & 8,79 & 0,00 & 1,10 & 100 \\
5 & 15,48 & 63,10 & 16,67 & 4,76 & 0,00 & 0,00 & 100 \\
6 & 11,54 & 64,10 & 19,23 & 3,85 & 1,28 & 0,00 & 100 \\
7 & 25,64 & 57,69 & 14,10 & 1,28 & 1,28 & 0,00 & 100 \\
8 & 26,74 & 60,47 & 10,47 & 0,00 & 2,33 & 0,00 & 100 \\
9 & 33,90 & 62,71 & 3,39 & 0,00 & 0,00 & 0,00 & 100 \\
10 & 60,87 & 26,09 & 8,70 & 4,35 & 0,00 & 0,00 & 100 \\
\hline Total & 33,20 & 50,06 & 11,54 & 3,37 & 1,17 & 0,65 & 100 \\
\hline
\end{tabular}

Se pude comprobar que también en este caso se observa muy poca movilidad. El cuadro 5 nos muestra que el 33,20\% de los hoteles no ha variado su posición en el ranking entre el máximo y el mínimo decil visitado. Este porcentaje es sensiblemente inferior al que presenta el indicador $\mathrm{IP}_{3}$ en el caso de hoteles que no han modificado su posición entre el momento inicial y final $(49,55 \%)$. Vemos que la distancia recorrida entre el punto máximo y mínimo para el $83,26 \%$ de los hoteles de la muestra ha sido como máximo de un decil. Este porcentaje llegaba al 88,07\% para la distancia entre el primer y último decil visitado. Esto nos vuelve a poner de manifiesto que hay algunos hoteles que si bien tienen cierto grado de movilidad, no se ve reflejada al final del 
periodo, en términos de subidas o bajadas en el ranking, lo que se podría interpretar como cierto efecto rebote por el cual un hotel tras subir o bajar en el ranking vuelve finalmente a su zona de partida.

El valor promedio de esta variable, al igual que en las anteriores es muy bajo $(0,91)$ y dado que la dispersión es también pequeña $(0,885)$, podemos considerar el valor promedio como representativo.

\section{CONCLUSIONES}

Aunque los conceptos de movilidad, rankings y TripAdvisor han sido estudiados por separado desde numerosos puntos de vista, y pese a ser ampliamente reconocida la influencia que los rankings (de hoteles o restaurantes) ejercen en el cliente a la hora de tomar la decisión de donde alojarse o donde ir a comer, son pocos los investigadores que han estudiado lo que hemos llamado la movilidad de los rankings, es decir, la variación de las posiciones que ocupan los distintos establecimientos en la clasificación a lo largo del tiempo.

Por eso el primer objetivo de esta investigación ha sido el de proponer una nueva línea de investigación en turismo apenas explorada hasta la fecha y centrada en el estudio de la movilidad en las clasificaciones de hoteles y restaurantes, principalmente en TripAdvisor y Booking.

Además, y siendo prioritario identificar las importantes implicaciones que la movilidad de estos rankings tiene para los distintos agentes en la industria turística, hemos analizado las consecuencias que un mayor o menor grado de movilidad puede tener para los clientes, hoteleros, inversores, instituciones y compañías responsables de diseñar estas influyentes clasificaciones, poniendo de manifiesto la complejidad de este concepto y los innumerables pros y contras que ofrece a cada uno de los actores implicados, siendo al mismo tiempo positiva para unos y negativa para otros.

Nuestro segundo objetivo ha sido el presentar los principales conceptos y definiciones para sentar las bases de futuros estudios en esta nueva materia. Así se han definido los conceptos de ranking, distinguiendo entre sólidos y líquidos, movilidad, desde una perspectiva macro y micro, y vector trayectoria.

Como tercer y último objetivo, nos propusimos crear un modelo para cuantificar la movilidad y analizar su evolución a lo largo del tiempo. Para ello se definieron cuatro indicadores parciales de movilidad que cuantifican varios aspectos de este concepto: variabilidad $\left(\mathrm{IP}_{1}\right)$, rachas $\left(\mathrm{IP}_{2}\right)$, distancia primer-último estado $\left(\mathrm{IP}_{3}\right)$ y distancia máximo-mínimo $\left(\mathrm{IP}_{4}\right)$ que se han calculado para una muestra de 771 hoteles de las seis primeras ciudades españolas por número de habitantes (Madrid, Barcelona, Valencia, Sevilla, Zaragoza y Málaga).

Todas las variables analizadas a partir de las trayectorias de los hoteles nos indican un grado de movilidad muy bajo. Así, el 85,99\% de los hoteles de la muestra presentan poca o nula variabilidad, $\left(\mathrm{IP}_{1} \leq 2\right)$, con una cifra promedio de 1,82 deciles distintos visitados a lo largo del periodo. En cuanto al número de rachas, el 66,92\% presentan un valor bajo o mínimo $\left(\mathrm{IP}_{2} \leq 2\right)$, con un promedio de 2,13 rachas para los hoteles analizados. Respecto a la variable $\mathrm{IP}_{3}$ (distancia primero-último), el $88,07 \%$ de los hoteles de la muestra pre- 
sentan valores muy pequeños $\left(\mathrm{IP}_{3} \leq 1\right)$, con un valor promedio de la distancia recorrida entre el momento inicial y el final de 0,68 deciles. Por último, el indicador $\mathrm{IP}_{4}$ (distancia máximo-mínimo), presenta unas cifras en consonancia con las otras variables, pues el $83,26 \%$ de los hoteles tiene valores muy pequeños o mínimos $\left(\mathrm{IP}_{4} \leq 1\right)$, con un valor promedio de 0,91 deciles.

Entre las contribuciones más destacadas de nuestra investigación, debemos destacar en primer lugar, el hecho de que este trabajo aporta una nueva aproximación teórica al concepto de movilidad, estableciendo las bases para el desarrollo de un marco teórico que permite inaugurar una nueva línea de investigación en Turismo, hasta ahora inexplorada, como es el de la movilidad de los rankings de hoteles y restaurantes. En este sentido, hemos contribuido con una novedosa metodología que permite medir la movilidad de un ranking de un modo eficiente, aplicada en este trabajo al ámbito concreto de los rankings de hoteles. El modelo conceptual elaborado, define el ranking como un sistema dinámico donde los distintos elementos que lo componen cambian de posición a lo largo del tiempo.

En segundo lugar, nuestra investigación pone de manifiesto la escasa movilidad que caracteriza a los rankings de hoteles de las seis mayores ciudades de España. Y, en tercer lugar, aunque no de menor importancia, el haber dado a conocer las importantes implicaciones que la mayor o menor movilidad de los rankings puede llegar a tener para empresarios hoteleros, inversores, empleados, directivos, clientes y destinos turísticos. La movilidad, debe ser considerada como una de las características que definen un ranking porque sus consecuencias para todos estos agentes son dignas de consideración.

Así, para los empresarios hoteleros de las ciudades analizadas, (todas con rankings sólidos, o de movilidad muy baja) y cuyos establecimientos se encuentren situados en la zona más alta de la clasificación, esta falta de movilidad les garantiza, con una elevada probabilidad, permanecer en esta privilegiada posición no solo a corto sino a medio plazo. Por el contrario, aquellos establecimientos situados en la zona media y baja del ranking, permanecerán en esas mismas zonas durante bastante tiempo.

En cuanto a los empleados y directivos, no tendrá sentido condicionar las políticas de personal (fijación de objetivos, promoción y ascensos, remuneraciones, bonus e incentivos, valoración del desempeño, etc.) a una mejora de la posición del hotel en el ranking, pues en estas ciudades, los cambios en la clasificación serán improbables e irrelevantes.

Para los inversores que tienen que decidir entre adquirir un hotel en funcionamiento o abrir uno nuevo, antes de tomar cualquier decisión, deberían conocer el grado de movilidad del ranking de la ciudad donde desean invertir, pues en ciudades como las analizadas, los hoteles de nueva creación tienen una gran oportunidad de situarse en la zona más alta del ranking en muy poco tiempo, como le ocurrió al hotel Room Mate Valeria en Málaga, alcanzando el puesto número 1 de la ciudad en apenas seis meses desde su inauguración.

Además, esta falta de movilidad en la clasificación debería influir en el precio de compraventa de establecimientos hoteleros, ya que se debería pagar una prima sobre dicho precio en hoteles situados en lo más alto del ranking, (pues seguirán en esa privilegiada zona con una alta probabilidad a medio plazo) o bien aplicar un descuento en el precio de adquisición de hoteles situados en las zonas media o baja (pues también seguirán en esas posiciones a medio plazo). 
Para los clientes, las implicaciones son también considerables. Un ranking líquido con cambios continuos en la clasificación (no es el caso de las ciudades que han sido objeto de nuestro estudio) puede generar cierta confusión e incertidumbre en el cliente viendo como aquel hotel que hace unas semanas estaba situado entre los mejores de la ciudad se encuentra ahora situado en los últimos puestos de la clasificación. Para las seis ciudades analizadas, los clientes percibirán cierta sensación de seguridad al comprobar que mes tras mes los hoteles elegidos por ellos permanecen en las mismas zonas del ranking.

Para los destinos turísticos, un ranking con gran movilidad podría estar reflejando una planta hotelera muy dinámica, con un alto grado de competitividad y niveles muy similares de calidad, no existiendo diferencias entre los distintos establecimientos, y siendo irrelevante la posición en un ranking donde los hoteles fluyen entre las posiciones altas, medias y bajas. Estaríamos entonces no ante un ranking propiamente dicho sino más bien ante un listado de hoteles todos de una calidad muy similar. En este sentido el concepto de movilidad en los rankings estaría relacionado con la desigualdad (en términos de calidad) de la planta hotelera. En las ciudades analizadas en nuestro estudio, la baja movilidad nos podría estar indicando que nos encontramos ante una planta hotelera con alto grado de desigualdad en la calidad con unos hoteles muy buenos siempre arriba y otros hoteles significativamente peores siempre abajo.

En definitiva, la movilidad de los rankings existe, aunque nadie la había medido hasta ahora. Nosotros lo hemos hecho por primera vez. Lo que no hemos querido hacer es juzgarla, es decir, considerarla como una característica positiva o negativa en sí misma. Como hemos visto, siempre encontraremos grupos interesados en que el ranking de hoteles o restaurantes de una ciudad sea sólido, inamovible. Pero también encontraremos en la misma ciudad colectivos interesados en que el ranking sea líquido. De ahí la complejidad de este concepto y los innumerables pros y contras que ofrece a cada uno de los agentes implicados. La movilidad será al mismo tiempo positiva para unos y negativa para otros.

Entre las limitaciones de nuestro estudio, que al mismo tiempo se convierten en oportunidades para futuros trabajos de investigación para todos aquellos interesados en este nuevo campo, debemos reconocer que sería conveniente ampliar la investigación a ciudades de otros países y continentes realizando comparativas de la movilidad de los rankings entre distintas zonas geográficas. También sería recomendable ampliar el horizonte temporal, y analizar un periodo de tiempo más amplio lo que permitiría comprobar si persiste la rigidez, o si por el contrario se aprecia algún tipo de tendencia creciente en la movilidad o incluso si aparecen signos de estacionalidad, con meses o trimestres donde la movilidad es significativamente distinta al resto.

También sería muy interesante aplicar el modelo a rankings desarrollados por otras webs (especialmente Booking.com), a otro tipo de establecimientos (restaurantes) o bien a ciudades con una tipología diferente de turismo (por ejemplo, destinos de sol y playa). Finalmente, y en este caso debido a las obvias limitaciones de espacio no hemos podido desarrollar el indicador parcial $\mathrm{IP}_{5}$, del que se habló en la sección 4.3 y que cuantifica el mayor salto en la posición que ha dado un hotel entre dos momentos consecutivos.

Por último, y tratándose de un trabajo introductorio, invitamos a jóvenes investigadores, así como a expertos en turismo y otras disciplinas a participar en este nuevo reto para aportar luz sobre unos instrumentos, los rankings, que han alcanzado una influencia inimaginable hace tan solo unos años. 


\section{BIBLIOGRAFÍA}

BABATIVA, G. y CORZO, J.A. (2010): "Propuesta de una prueba de rachas recortada para hipótesis de simetría". Revista Colombiana de Estadística, 33(251), 271.

BIFFARO, L. (2015): "Unfair Commercial Practices and Online Consumer Reviews: the Italian TripAdvisor Case". Rivista Italiana di Antitrust/Italian Antitrust Review, 2 (1).

BUELA-CASAL, G., GUTIÉRREZ-MARTÍNEZ, O., BERMÚDEZ-SÁNCHEZ, M.P. y VADILLO-MUÑOZ, O. (2007): "Comparative study of international academic rankings of universities". Scientometrics, 71 (3), 349-365.

CALLARISA-FIOL, L.J., SÁNCHEZ-GARCÍA, J., MOLINER TENA, M.Á. y FORGAS-COLL, S. (2012): "La importancia de las comunidades virtuales para el análisis del valor de marca. El caso de TripAdvisor en Hong Kong y París". Papers de Turisme, 54, 89-115.

CHEN, Y.F. y LAW, R. (2016): "A Review of Research on Electronic Word-of-Mouth in Hospitality and Tourism Management”. International Journal of Hospitality \& Tourism Administration, 17 (4), 347-372.

DEEG, D.J. (2002): “Attrition in longitudinal population studies: Does it affect the generalizability of the findings?”. Journal of Clinical Epidemiology, 55 (3), 213-215.

DRISCOLL, A., LAWSON, R. y NIVEN, B. (1994): “Measuring tourists' destination perceptions". Annals of Tourism Research, 21 (3), 499-511.

DURÁN, P. (2005): "Los datos perdidos en estudios de investigación ¿son realmente datos perdidos?". Archivos argentinos de pediatría, 103 (6), 566-568.

EE KIM, S. y LEHTO, X.Y. (2012): “The voice of tourists with mobility disabilities: insights from online customer complaint websites". International Journal of Contemporary Hospitality Management, 24 (3), 451-476.

ENGELS, J.M. y DIEHR, P. (2003): "Imputation of missing longitudinal data: a comparison of methods». Journal of Clinical Epidemiology, 56 (10), 968-976.

FERNÁNDEZ-MORALES, A. (2017): “Tourism mobility in time and seasonality in tourism". Rivista Italiana di Economia Demografia e Statistica, LXXI (2), 35-52.

FERRANTE, M., DE CANTIS, S. y SHOVAL, N. (2018): “A general framework for collecting and analysing the tracking data of cruise passengers at the destination". Current Issues in Tourism, 21 (12), 1.426-1.451.

FERREIRA, R.R., DEFRANCO, A.L. y RAPPOLE, C.L. (1994): "Rating the hospitality journals". International Journal of Hospitality Management, 13 (3), 209-218.

FIELDS, G. S. (2000): Income mobility: Concepts and measures. Cornell University ILR School.

FU, J.C., y KOUTRAS, M.V. (1994): "Distribution theory of runs: a Markov chain approach». Journal of the American Statistical Association, 89 (427), 1.050-1.058.

GHOSE, A., IPEIROTIS, P.G. y LI, B. (2012): "Designing Ranking Systems for Hotels on Travel Search Engines by Mining User-Generated and Crowdsourced Content". Marketing Science, 31 (3), 493-520.

GOODMAN, L.A. (1969): "How to ransack social mobility tables and other kinds of cross-classification tables". American Journal of Sociology, 75 (1), 1-40. 
GRETZEL, U., HYAN-YOO, K. y PURIFOY, M. (2007): Online Travel Review Study: Role and Impact of Online Travel Reviews. Laboratory for Intelligent Systems in Tourism, Texas, A\&M University.

HALL, C. M. (2005):Tourism: Rethinking the social science of mobility. Pearson Education. HENSENS, W. (2010): "The reliability of data from guest reviews on TripAdvisor as a contemporary social media platform”. En SAIMS Annual Conference, 12-15.

HUNTER, L.C. y REID, G.L. (1968): Urban worker mobility (Vol. 5). Organisation for Economic Co-operation and Development.

JEONG, I. (2003): Interregional Labor Mobility.

JURCA, R., GARCIN, F., TALWAR, A. y FALTINGS, B. (2010): "Reporting incentives and biases in online review forums". ACM Transactions on the Web, 4 (2), 1-27.

KWOK, L., XIE, K. y TORI, R. (2017): "Thematic framework of online review research: A systematic analysis of contemporary literature on seven major hospitality and tourism journals». International Journal of Contemporary Hospitality Management, 29 (1), 307-354.

LÖFGREN, O. (2015): "Modes and Moods of Mobility: Tourists and Commuters". Culture Unbound: Journal of Current Cultural Research, 7 (2), 175-195.

MATEOS, J.C., MARTÍN, M.Á.R. y MARTÍN-VELICIA, F.A. (2014). Disrupción en el uso de la web 2.0 en los hoteles independientes. En Cultura, desarrollo y nuevas tecnologías: VII Jornadas de Investigación en Turismo, Sevilla, Universidad de Sevilla, 169-183.

MELIÁN GONZÁLEZ, S., BULCHAND GIDUMAL, J. y GONZÁLEZ LÓPEZVALCÁRCEL, B. (2010): «La participación de los clientes en sitios web de valoración de servicios turístico. El caso de TripAdvisor». Revista de Análisis Turístico,10 , 17-22.

MELLINAS, J.P., MARÍA-DOLORES, S.M.M. y GARCÍA, J.J.B. (2015): “Booking. com: The unexpected scoring system". Tourism Management, 49, 72-74.

MOLINILLO, S., XIMÉNEZ-DE-SANDOVAL, J.L., FERMÁNDEZ-MORALES, A. y COCA-STEFANIAK, A. (2016): "Hotel assessment through social media-TripAdvisor as a case study". Tourism \& Management Studies, 12 (1),15-24.

NICHOLSON, A.J. (1986): “The randomness of accident counts". Accident Analysis \& Prevention, 18 (3), 193-198.

O'BRIEN, P. C. y DYCK, P.J. (1985): “A runs test based on run lengths". Biometrics, 237-244.

ONG, B.S. (2012): "The Perceived Influence of User Reviews in the Hospitality Industry". Journal of Hospitality Marketing \& Management, 21(5), 463-485.

OTT, M., CARDIE, C. y HANCOCK, J. (2012). Estimating the Prevalence of Deception in Online Review Communities. En Proceedings of the 21st international conference on WWW 12, ACM, 201-210.

PRAIS, S. (1955): "Measuring social mobility". Journal of the Royal Statistical Society. Series A (General), 118 (1), 56-66.

RYAN, C. (2005): "The ranking and rating of academics and journals in tourism research". Tourism Management, 26 (5), 657-662.

SALAMANCA, J.A.C., MORALES, M.E.V. y MÁRQUEZ, J.G.B. (2017): “Una prueba de rachas para la alternativa" estocásticamente mayor que" en muestras de la distribución lognormal”. Comunicaciones en Estadística, 10 (2), 297-314. 
SASSEN, S. (1990): The mobility of labor and capital: A study in international investment and labor flow. Cambridge University Press.

SCOTT, S.V, y ORLIKOWSKI, W.J. (2012): "Reconfiguring relations of accountability: Materialization of social media in the travel sector". Accounting, Organizations and Society, 37 (1), 26-40.

SHELDON, P.J. (1991): “An authorship analysis of tourism research”. Annals of Tourism Research, 18 (3), 473-484.

SHOVAL, N. y ISAACSON, M. (2009): Tourist mobility and advanced tracking technologies. Routledge.

SMYTH, B., WU, G. y GREENE, D. (2010): “Does TripAdvisor Makes Hotels Better ?”. Technical Report, (08).

SOTIRIADIS, M.D. y SOTIRIADIS, M.D. (2017): "Sharing tourism experiences in social media: A literature review and a set of suggested business strategies". International Journal of Contemporary Hospitality Management, 29 (1), 179-225.

TRIPADVISOR. (2016): Changes to the TripAdvisor Popularity Ranking Algorithm. https://www.TripAdvisor.com/TripAdvisorInsights/n2701/changes-TripAdvisor-popularity-ranking-algorithm

TWISK, J. y DE VENTE, W. (2002): “Attrition in longitudinal studies: how to deal with missing data". Journal of clinical epidemiology, 55 (4), 329-337.

VERMA, R., STOCK, D. y MCCARTHY, L. (2012): “Customer Preferences for Online, Social Media, and Mobile Innovations in the Hospitality Industry". Cornell Hospitality Quarterly, 53(3), 183-186.

XIANG, Z. y GRETZEL, U. (2010): "Role of social media in online travel information search". Tourism Management, 31(2), 179-188. 